\title{
Folded superstructure and degeneracy-enhanced band gap in the weak-coupling charge density wave system $2 \mathrm{H}$-TaSe
}

\author{
Y. W. Li, ${ }^{1}$ J. Jiang, ${ }^{2,3}$ H. F. Yang, ${ }^{2}$ D. Prabhakaran, ${ }^{1}$ Z. K. Liu, ${ }^{2}$ L. X. Yang,,${ }^{4, *}$ and Y. L. Chen ${ }^{1,2,4, \dagger}$ \\ ${ }^{1}$ Department of Physics, University of Oxford, Oxford, OX1 3PU, United Kingdom \\ ${ }^{2}$ School of Physical Science and Technology, ShanghaiTech University and CAS-Shanghai Science Research Center, Shanghai 201210, \\ People's Republic of China \\ ${ }^{3}$ Advanced Light Source, Lawrence Berkeley National Laboratory, Berkeley, California 94720, USA \\ ${ }^{4}$ State Key Laboratory of Low Dimensional Quantum Physics, Department of Physics and Collaborative Innovation Center of Quantum \\ Matter, Tsinghua University, Beijing 100084, People's Republic of China
}

(Received 23 August 2017; published 9 March 2018)

\begin{abstract}
Using high-resolution angle-resolved photoemission spectroscopy (ARPES), we have mapped out the reconstructed electronic structure in the commensurate charge-density-wave (CDW) state of quasi-two-dimensional transition metal dichalcogenide $2 \mathrm{H}-\mathrm{TaSe}_{2}$. The observation of the fine structure near Brillouin zone (BZ) center supplements the picture of Fermi surface folding in the $3 \times 3 \mathrm{CDW}$ state. In addition to the anisotropic CDW band gaps that energetically stabilize the system at the Fermi level in the first-order lock-in transition, we found band reconstruction at high binding energy, which can be well explained by the hybridization between main bands (MBs) and folded bands (FBs). Furthermore, in contrast to the perfectly nested quasi-one-dimensional system, triple-nesting-vector-induced CDW FBs increase the degeneracy of the band crossing and thus further enlarge the magnitude of band gap at certain momentum-energy positions. The visualization and modeling of CDW gaps in momentum-energy space reconciles the long-lasting controversy on the gap magnitude and suggests a weak-coupling Peierls physics in this system.
\end{abstract}

DOI: 10.1103/PhysRevB.97.115118

\section{INTRODUCTION}

Charge-density waves (CDWs) have been discovered in quasi-two-dimensional (quasi-2D) transition metal dichalcogenides (TMDs) for decades [1-3]. However, two fundamental questions remain unsolved from an electronic structure perspective [4]: (1) what influence do CDWs have on the electronlattice system, or how does electronic band reconstruct in a CDW phase? (2) What is the driving force of the CDW transition? For the first question, pseudogap states have been reported near normal-incommensurate transition temperature [5-7]. In commensurate regime, Fermi surface (FS) folding, band folding, CDW gap opening, and mysterious high-energy "kinks" have been observed by angle-resolved photoemission spectroscopy (ARPES) [8-11]. However, there still exists controversy about the most fundamental parameter, the size of CDW gap obtained by different experimental approaches (including ARPES [5,10,12,13], scanning tunneling spectroscopy [14], and optical spectroscopy [15]), which calls for further exploration. For the second question, the leading candidate model for CDW mechanism, FS nesting (Peierls instability indicated by a divergence of electronic susceptibility) faces constant questioning in quasi-2D systems [16,17]. Unlike quasi-1D cases in which nesting criterion is reasonably met due to perfectly parallel FS sheets [18], FS topology is much more complicated and hence weak-nested in quasi-2D CDW systems [16]. To make this model solid, more effects including electron-

\footnotetext{
*1xyang@tsinghua.edu.cn

†Yulin.Chen@physics.ox.ac.uk
}

electron interaction [11] and momentum-dependent electronphonon coupling $[17,19,20]$ are introduced. Some researchers imply that $2 \mathrm{D}$ systems might fall in the strong-coupling regime $[4,21]$ where the classical Peierls weak-coupling mean-field model [21-23] fails. Besides, other mechanisms such as Fermi patch nesting [24], saddle point nesting [25], and lattice-driven [26] models are proposed. In short, there is still intense controversy on electronic band reconstruction in CDW phase and essential lack of a standard model for CDW formation in quasi-2D TMDs.

$2 \mathrm{H}-\mathrm{TaSe}_{2}$ exhibits a second-order transition from normal phase to near commensurate $3 \times 3$ ordered phase at $122 \mathrm{~K}$ followed by a first-order lock-in transition to the commensurate $3 \times 3$ ordered phase at $90 \mathrm{~K}$ [4] upon cooling. With virtually the highest commensurate CDW onset temperature in $2 \mathrm{H}$-TMD family [27], electronic band reconstruction in $2 \mathrm{H}-\mathrm{TaSe}_{2}$ is reasonably strong for observation. Moreover, compared to other classical TMD systems such as Mott insulator $1 \mathrm{~T}-\mathrm{TaS}_{2}$ [11] and superconducting $2 \mathrm{H}-\mathrm{NbSe}_{2}$ [9], the phase diagram of $2 \mathrm{H}-\mathrm{TaSe}_{2}$ is less complex. Mott state caused by electronelectron interaction is excluded. The superconducting transition at an extremely low temperature of $0.15 \mathrm{~K}$ [27] indicates minimal competition against CDWs. Thus, $2 \mathrm{H}-\mathrm{TaSe}_{2}$ is a relatively pure and canonical system for 2D-CDW measurement and a touchstone for modeling.

Although intensive research has been done on $2 H-\mathrm{TaSe}_{2}$ in the past decades, there still lacks consistency between various experimental results with existing theories. On one hand, electronic structure of $2 \mathrm{H}-\mathrm{TaSe}_{2}$ detected by ARPES near the Fermi level shows clear gap opening and backfolding features in the CDW phase $[4,5,10]$. From temperature-dependent 
measurement [7,28], superstructure order and FS nesting vector show consistent evolution. These results strongly favor the conventional nesting instability to be the major driven force of CDW phase although part of the folded bands (FBs) remains experimentally elusive [4,5]. On the other hand, theorists believe that Peierls nested construction is mathematically fragile to apply to TMD systems [17]. Despite the argument on the driving mechanism, an extreme uncertainty of magnitude of CDW gap in $2 H-\mathrm{TaSe}_{2}$ from 24 to $160 \mathrm{meV}$ [10,12-15] makes it an ambiguous criterion for theory screening.

In this article, we report a direct observation of the fine structure of FBs near $\bar{\Gamma}$ which is missing in the previous ARPES study in the CDW phase of the $2 \mathrm{H}-\mathrm{TaSe}_{2}$. CDW band gap with precise momentum-energy distribution has been revealed. The trace of CDW gaps matches well with the band crossings between the corresponding main band (MB) and FBs in the noninteracting $3 \times 3$ ordered picture. Our comprehensive experimental results and analysis validate the band hybridization picture suggested in previous studies $[4,10]$. Beyond that, a strong correlation between the degeneracy of band crossings and the magnitude of the gap is experimentally confirmed by a dramatic enhancement of gap value at corresponding momentum. This information not only helps us resolve the controversy on the magnitude of CDW gap in this specific material, considering its simple phase diagram and the fine CDW band structure experimentally observed, but also provides a benchmark for quasi-2D CDW theories.

\section{METHODS}

$2 \mathrm{H}-\mathrm{TaSe}_{2}$ single crystals are grown from Ta metal and Se by iodine vapor transport [29]. ARPES measurements are performed at beam line I05 (Diamond Light Source, UK) equipped with an R4000 electron analyzer. The overall energy and angular resolutions are $10 \mathrm{meV}$ and $0.2^{\circ}$, respectively. The samples are cleaved in situ along (001) direction. Normal and CDW phases are measured at $130 \mathrm{~K}$ and $10 \mathrm{~K}$, respectively. All calculated band structures in this paper are fitted to experimental data via a conventional tight-binding (TB) model $[9,10,16,30]$. Details of this technique and parameters of the model can be found in the Supplemental Material SI A [31]. CDW band gaps are pinpointed in momentum-energy space via maximum-intensity-projection (MIP) method. Principles and details of this approach can be found in the Supplemental Material SI C [31].

\section{RESULTS}

\section{A. Band structure reconstruction in CDW phase}

In the normal phase of $2 \mathrm{H}-\mathrm{TaSe}_{2}$, the atomic coordination in the real space and electronic structure in the reciprocal space are shown in Figs. 1(a) and 1(b), respectively. The electronic structure in the normal phase shows a clear semimetal nature. The FS consists of circular hole-pockets centered at $\bar{\Gamma}$ and $\overline{\mathrm{K}}$, and "dog-bone" electron-pockets centered at $\overline{\mathrm{M}}$ [see Fig. 1(b)]. The electron- and hole-pockets are from two different band sheets. The experimental band dispersion agrees well with previous ARPES study $[4,5,10]$ and recent ab initio calculation [32], though in which $E_{F}$ is slightly overestimated by $50 \mathrm{meV}$. The semimetal FS topology also originates directly from its
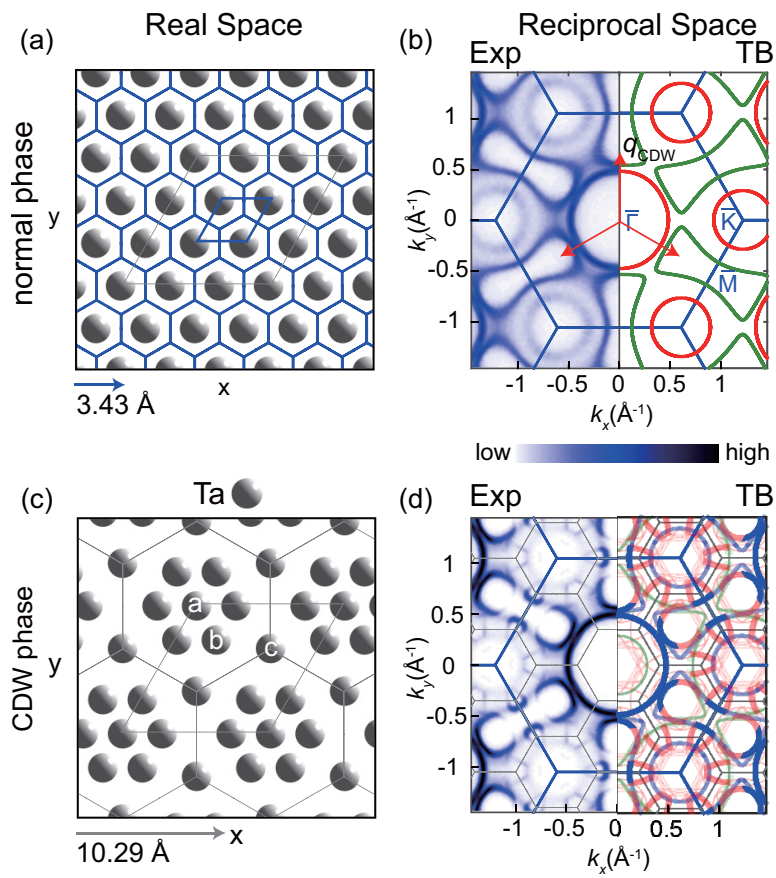

FIG. 1. Super-lattice formation and FS reconstruction in CDW phase transition. (a) Ta layer atomic arrangement in the normal phase. Wigner-Seitz (hexagon) and conventional (rhombus) unit cells are appended with blue lines. (b) Electronic band structure at Fermi level consists of hole-pockets (red lines) centered at $\bar{\Gamma}$ and $\overline{\mathrm{K}}$, and electron-pockets (green lines) centered at $\overline{\mathrm{M}}$. TB calculation (right panel) nicely reproduce ARPES data (left panel). (c) Same as (a) but in CDW phase. Each $3 \times 3$ super-lattice contains one $\mathrm{Ta}_{\mathrm{a}}$, six $\mathrm{Ta}_{\mathrm{b}}$, and two $\mathrm{Ta}_{\mathrm{c}}$ atoms. The neighboring six $\mathrm{Ta}_{\mathrm{b}}$ atoms move towards the center $\mathrm{Ta}_{\mathrm{a}}$, whereas the $\mathrm{Ta}_{\mathrm{c}}$ atom positions remain unchanged during the phase transition. Note that the atomic displacement in CDW phase is exaggerated for better illustration. The new unit cells under the tripled translation vector (the gray arrow) are appended with gray lines. (d) Same as (b) but in CDW phase. TB calculation (right panel) based on $3 \times 3$ FS folding (details and principle can be found in the Supplemental Material SI B [31]) can well explain the ARPES result (left panel). The MBs, first- and second-order FBs are marked by blue, red, and green lines, respectively, in the calculation. The thickness of lines indicates spectral weight enhancement due to pronounced matrix element effect. BZs of normal phase and new mini-BZs of CDW phase are appended with blue and gray lines in (b) and (d). The triple CDW nesting vectors $q=\frac{2}{3} \bar{\Gamma} \bar{M}$ are indicated by red arrow in (b).

chemical configuration: the Ta-Se bond requires four electrons from each Ta atom since the oxidation state of Ta and Se are +4 and -2 , respectively. Considering the outermost electron shell of Ta atoms is $6 s^{2} 5 d^{3}$, there is one $5 d$ conducting electron left for each site (two electrons for a unit cell) half-filling the two band sheets at Fermi level [33].

The CDW in $2 \mathrm{H}-\mathrm{TaSe}_{2}$ causes a significant reconstruction of atomic coordination in the real space [Fig. 1(c)] as well as electronic band structure in the reciprocal space [Fig. 1(d)]. In the real space, six $\mathrm{Ta}_{\mathrm{b}}$ atoms move towards the center $\mathrm{Ta}_{\mathrm{a}}$ atom, forming a seven-atom-cluster, while the $\mathrm{Ta}_{\mathrm{c}}$ positions remain unchanged [4]. In the reciprocal space, in agreement with early studies $[4,5,10]$, the $\overline{\mathrm{K}}$-centered hole-pocket completely disappears whereas the $\overline{\mathrm{M}}$-centered electron-pocket is partially 

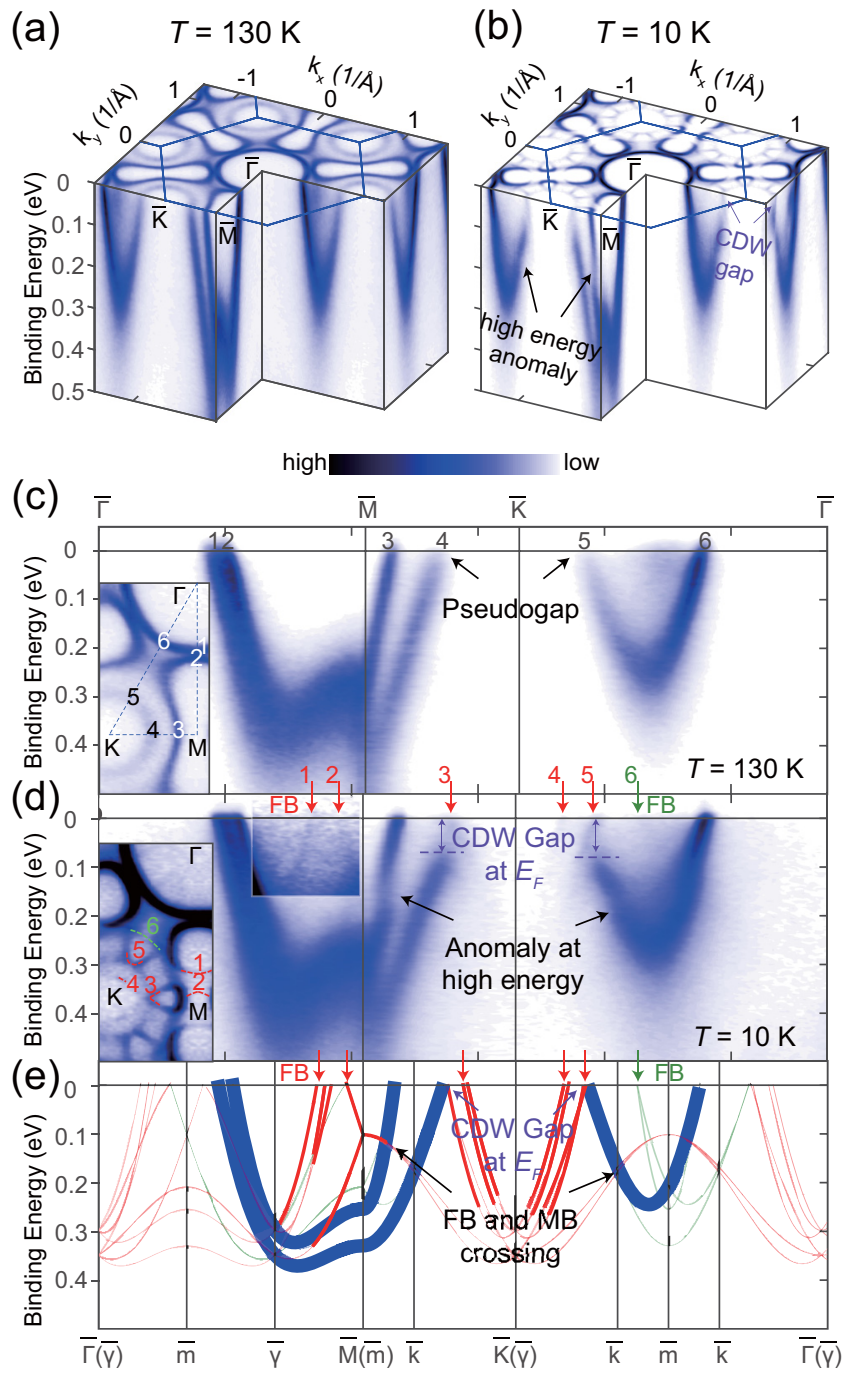

FIG. 2. Band structure reconstruction in $3 \times 3 \mathrm{CDW}$ phase. (a), (b) 3D intensity plot of ARPES data using photon energy $80 \mathrm{eV}$ measured in normal phase at $130 \mathrm{~K}$ (a) and in CDW phase at $10 \mathrm{~K}$ (b). (c)-(e) Comparison among experimental band dispersion in normal phase (c), CDW phase (d), and calculation (e) based on TB fitting and FS folding scheme along high symmetry direction $\bar{\Gamma}-\overline{\mathrm{K}}-\overline{\mathrm{M}}-\bar{\Gamma}$. In (c), (d) the insets show corresponding ARPES FS maps. Features at $E_{F}$ are marked correspondingly. In (e) MBs, first- and second-order FBs are marked with blue, red, and green lines, respectively. The thickness of lines in TB fitting indicates spectral weight enhancement due to pronounced matrix element effect.

gapped, forming triangular pockets on the FS. The validity of $3 \times 3$ FS folding (details of this model can be found in the Supplemental Material SI B [31]) is strengthened as all these experimental features have their counterparts in the calculation [Fig. 1(d)].

A general comparison of band structure between normal and CDW phase is presented in Fig. 2. As shown in the 3D intensity plots [Figs. 2(a) and 2(b)], the band reconstruction in the CDW phase happens not only near $E_{F}$, but also at high binding energies, as marked in Fig. 2(b). In consistence with previous report [5], we observed clear pseudogaps at $\overline{\mathrm{K}}$-centered holepockets [see Fig. 2(c)] marked by the suppression of spectral weight near $E_{F}$ (more details can be found in Supplemental
Material SI D [31]). In the CDW state, we observe clear CDW gap as large as $70 \mathrm{meV}$ at the Fermi crossing of the $\overline{\mathrm{K}}$-centered pockets. We also notice some anomalies of band dispersions at high binding energy [see Fig. 2(d)]. The momentum positions of these high energy anomalies match exactly with the band crossings between the $\mathrm{MB}$ and $\mathrm{FBs}$ as predicted by $\mathrm{TB}$ calculation [see Fig. 2(e)]. However, the fine structure of the FBs around $\bar{\Gamma}$ is very weak with this measured photon energy $(80 \mathrm{eV})$ in Fig. 2, which is probably due to the matrix element effect induced by the weak CDW superpotential [30]. To see the fine structure of the FBs clearly, we switch to another photon energy $(50 \mathrm{eV})$ in the following, where the intensity around $\bar{\Gamma}$ is significantly enhanced (the possibility of the contribution of the $k_{z}$ effect has been ruled out as discussed in Supplemental Material SI F [31]).

\section{B. CDW FBs and new translational symmetry}

Here, we provide an equivalent interpretation for CDW band reconstruction as FS folding but from a distinct perspective to emphasize the new translational symmetry. As indicated in the upper panel of Fig. 3(a), if all bands (MBs plus FBs) in CDW phase are included regardless of the prominent matrix element effect, then the FS should have the corresponding symmetry of the mini-BZs (new Brillouin zone based on the tripled translational vector in CDW phase). Take two mini-BZs, miniBZ 1 marked magenta centered at $\bar{\Gamma}$ and mini-BZ 2 marked green next to it, as examples. They are indistinguishable as linked by a CDW reciprocal vector $q=\frac{2}{3} \bar{\Gamma} \bar{M}$ but totally different in the normal phase. When the system evolves into the CDW phase, identical band dispersions form in both mini-BZs. However, their intensities are "rendered" unevenly as if they are in a "background" of different MBs. Mini-BZ 1 has a "blank background," so it is rendered by pure CDW FB features in the low-temperature phase [see the upper panel of Fig. 3(b)]. The direct observation of fine folded superstructure without any interference of MBs provides compelling experimental evidence for FS folding scheme under $3 \times 3$ ordering. In contrast, mini-BZ 2 is under an MB "background" with mirror symmetry hence its CDW spectrum shows a weak FB feature with a six-fold rotational symmetry appended on top [see the lower panel of Fig. 3(b)].

This perspective is further strengthened by a detailed comparison of band dispersions between the CDW phase and the normal phase in mini-BZ 2 [Figs. 3(c)-3(f)]. Before the new symmetry is introduced, high symmetric points, $\bar{m}_{i}(i=1,2,3,4)$ and $\bar{k}_{j}(j=1,2,3)$ are inequivalent in momentum space hence corresponding band dispersions vary [see Figs. 3(c) and 3(d)]. The formation of FBs under CDW superpotential compensates for this difference in the CDW state [see Figs. 3(e) and 3(f)], making the dispersions near $\bar{m}_{i}$ (and $\bar{k}_{j}$ ) equivalent except for intensity difference. The emergence of FBs is revealed by ARPES intensity plots [Figs. 3(e) and $3(\mathrm{f})$ ] as well as corresponding peaks in momentum distribution curve (MDC) plots [Figs. 3(i) and 3(j)].

Based on this understanding, the mini-BZ becomes the building block with all the information on electronic structure in CDW regime. The FS in a single mini-BZ consists of concentric multi-walled $\bar{\gamma}$-centered electron-pockets and double-walled $\bar{k}$-centered electron-pockets (see Fig. 4). We 
(a)

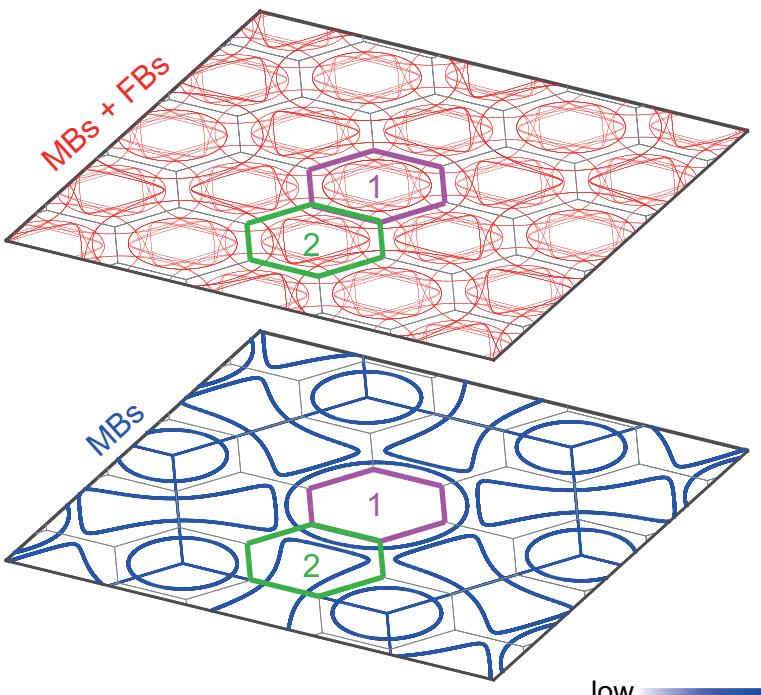

(c)
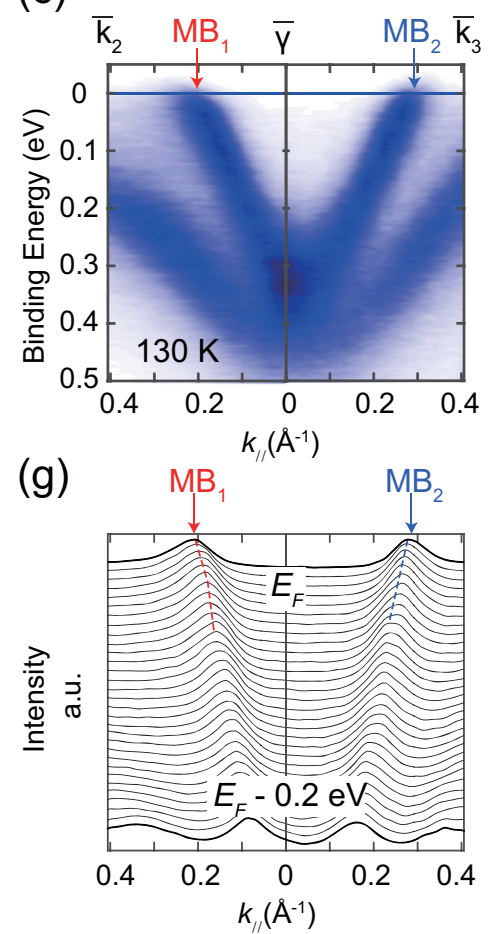

(d)

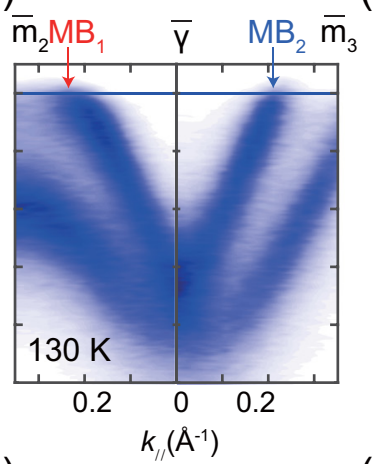

(h)

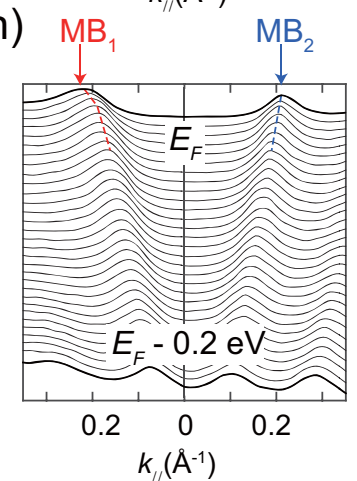

(b)

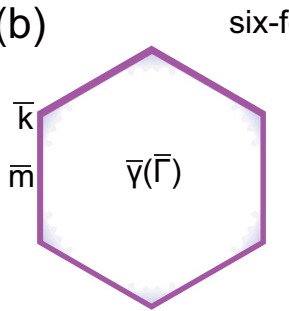

six-fold symmetric CDW FBs

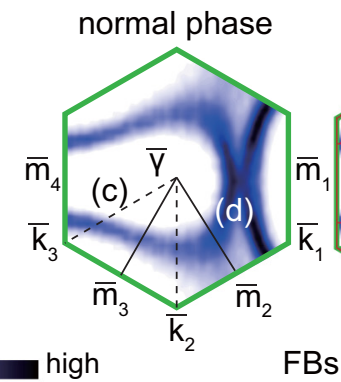

(e)

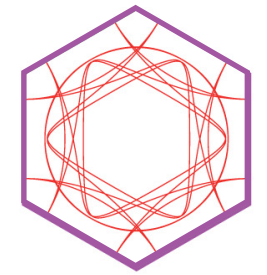

normal phase
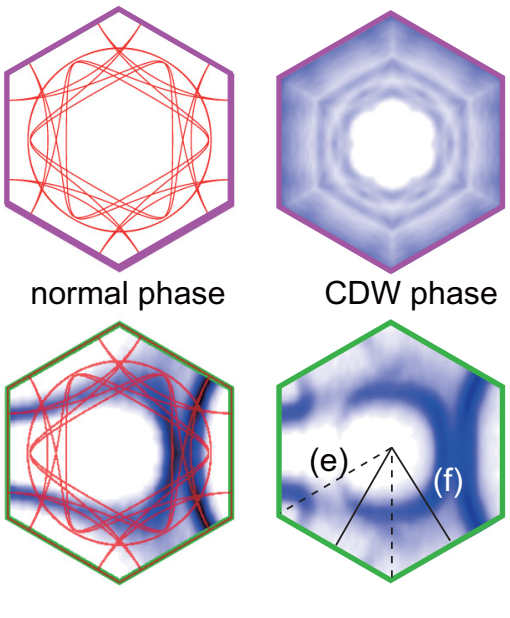

FBs hybridized with MBs

(f)


(i)
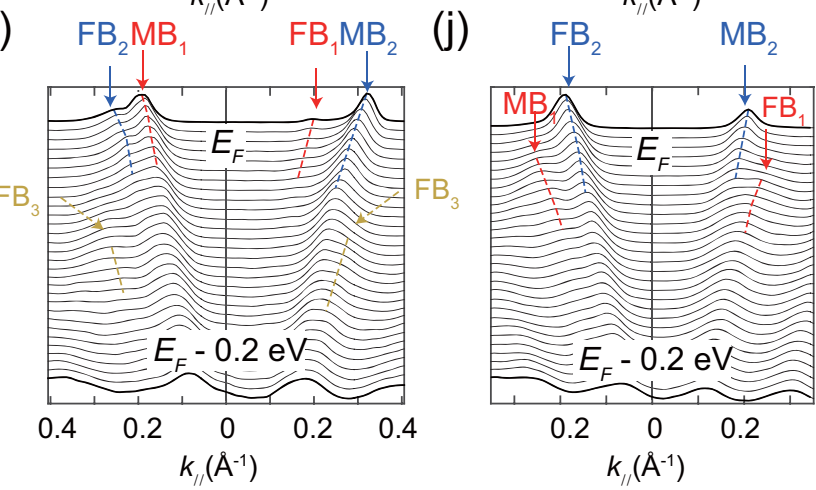

FIG. 3. Weak FBs features with new periodicity on top of MBs. (a) Schematic of FS of MBs (lower) and overall bands (MBs + FBs) in the CDW phase (upper). Original BZs and the new mini-BZs are marked by blue and gray lines. The two-bold mini-BZs (1, magenta; 2, green) are selected to present how FBs interact with MBs in (b). (b) Pure folded features with six-fold rotational symmetry (upper row) and folded features under the influence of MBs (lower row) in mini-BZ1 and mini-BZ2 indicated in (a), respectively. (c), (d) Normal phase experimental band dispersion along $\bar{k}_{2}-\bar{\gamma}-\bar{k}_{3}$ (c) and $\bar{m}_{2}-\bar{\gamma}-\bar{m}_{3}$ (d) in mini-BZ2 indicated in (b). (e), (f) Same as (c), (d) but in CDW phase. (g)-(j) MDC plots of (c)-(f) in an energy window from $E_{F}$ to $E_{F}-0.2 \mathrm{eV}$.

note that the sign of Hall coefficient reverses [6] after CDW phase transition, which may be related to the annihilation of hole-pockets on the FS.

\section{CDW gaps and nesting degeneracy}

Our systematic ARPES study has confirmed the validity of FS folding scheme on electronic band structure reconstruction in the CDW regime. To some extent, the first question on electronic structure reconstruction in CDW phase raised in the beginning of the manuscript is answered. However, why this state is energetically favored remains elusive. Borisenko et al. [5] have suggested that energy gain, marked by the formation of pseudogaps at $\overline{\mathrm{K}}$-centered hole-pockets, occurs near $\mathrm{CDW}$ onset transition at $122 \mathrm{~K}$, despite that the normal to incommensurate CDW transition is second order with no latent heat associated. However, the lock-in transition around $90 \mathrm{~K}$ is firstorder supported by a hysteresis on specific heat [34], we thus argue that discontinuous energy drop of electron-lattice system should occur when commensurate CDW FBs are formed and 
(a)
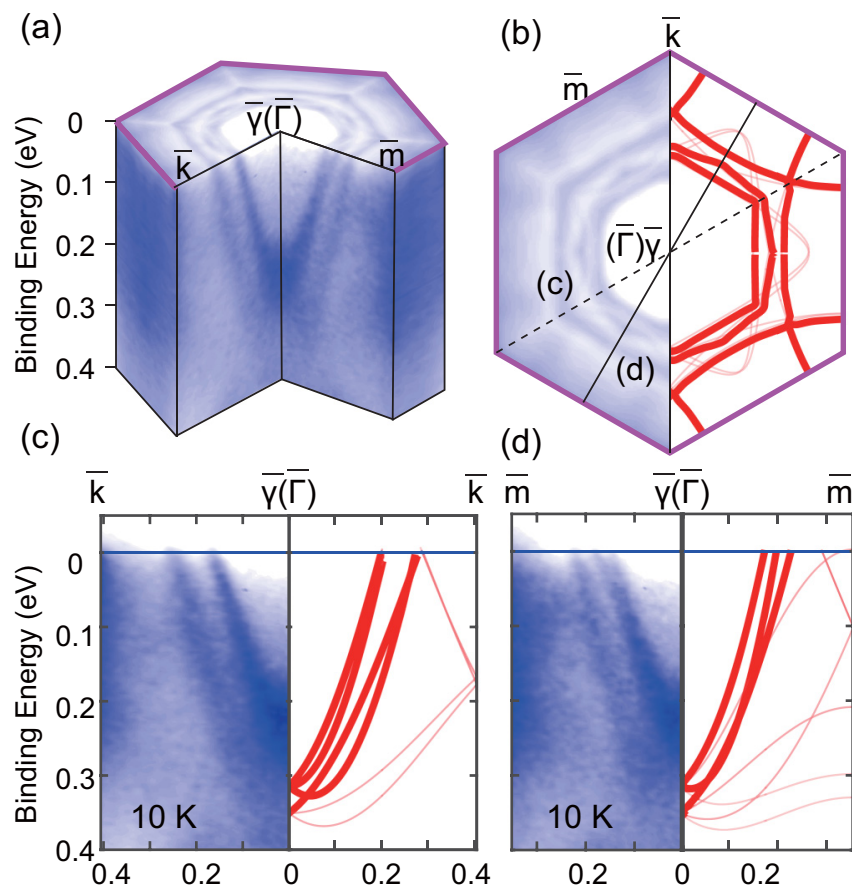

(e)
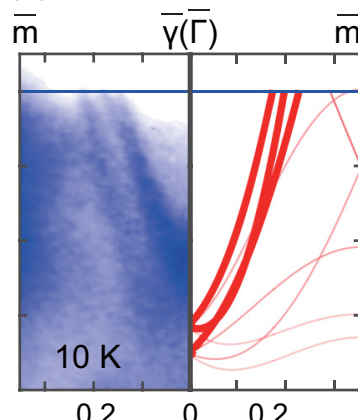

(f)
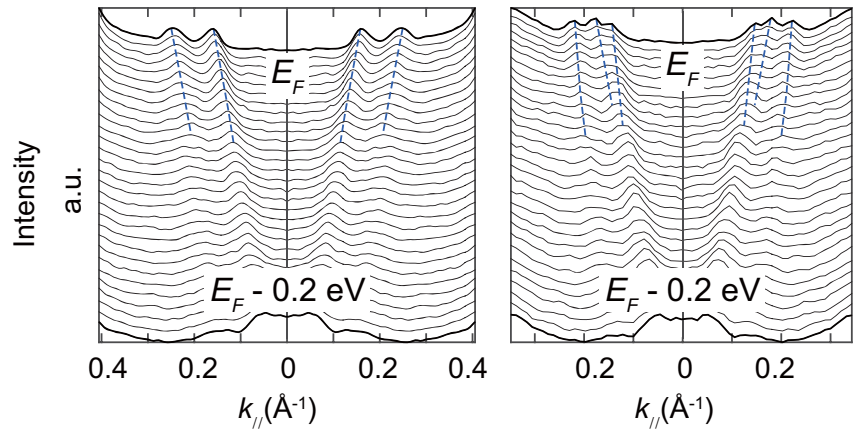

FIG. 4. Pure folded features at $\bar{\Gamma}$. (a) 3D intensity plot of ARPES data of mini-BZ1 [the magenta hexagon shown in Fig. 3(a)] in CDW phase. (b) Zoomed in Fermi surface around $\bar{\Gamma}$ showing pure folded features. Comparison shows an excellent agreement between experimental $E_{F}$ contour (left) and corresponding simulation based on TB fitting and FS folding (right). (c), (d) Comparisons show an excellent agreement between experimental CDW band dispersion and corresponding simulation based on TB fitting and FS folding along $\bar{k}-\bar{\gamma}(\bar{\Gamma})-\bar{k}(\mathrm{c})$ and $\bar{m}-\bar{\gamma}(\bar{\Gamma})-\bar{m}$ (d). The small mismatch between experiment and calculation of the binding energy of band bottom at $\bar{\Gamma}$ might be a $k_{z}$ effect (discussions can be found in Supplemental Material SI F [31]). (e), (f) MDC plots of (c), (d) in an energy window from $E_{F}$ to $E_{F}-0.2 \mathrm{eV}$.

band gaps are created near $E_{F}$. The observed pseudogap [see Fig. 2(c)], on the other hand, may originate from precursor fluctuations of the CDW order in the normal state [5,28].

Identification and positioning of CDW gap in the momentum space has never been a trivial process [4]. We notice that a dip in the local density of states (LDOS) is created at the energy positions where the band gap is formed under periodic potential. Similarly, CDW gaps should also suppress the spectral weight of the MB along energy and leave a local dip in the intensity map. Based on this idea, we have developed a method using the dip trace in the maximum intensity projection
(MIP) map (see more details in the Supplemental Material SI C [31]) to track the momentum distribution of CDW gap. 2D momentum distributions of the CDW gap candidates are illustrated in the broad MIP [Fig. 5(d)] and three zoomed-in areas [Figs. 5(e)-5(g)], which show excellent agreement with the intersection line between MB and FB sheets based on TB model in Figs. 5(a)-5(c). We thus suggest that CDW gaps are caused by hybridization between MBs and FBs.

To further visualize these band gaps, band dispersions along the gap positions are presented in comparison with high-temperature counterparts (Fig. 6). We have proved that MIP method not only precisely locate the CDW gaps in the momentum space, but also render gap values that are consistent with ones measured by the conventional method [see Supplemental Material SI C [31]]. Most of the expected gaps (e.g., A-B-C [Fig. 6(a)] and D-E [Fig. 6(b)]) are resolved, whereas others only render broad features (e.g., I-J [Fig. 6(d)]) due to finite energy resolution and broadening effects. Both two MB sheets forming the electron- and hole-pockets in the normal phase open CDW gaps with intricate momentumenergy distribution over the whole $\mathrm{BZ}$ in an energy window over $0.2 \mathrm{eV}$. The gap which completely destroys the $\overline{\mathrm{K}}$-centered hole-pocket at $E_{F}$ is an extension of D-E [see Figs. 5(b), 5(f), and $6(\mathrm{~b})]$, and the one which partially gaps the $\bar{M}$-centered electron-pocket is an extension of L-J and I-J below $E_{F}$ [see Figs. 5(c), 5(g), and 6(d)]. Direct volume visualization of CDW gap reconstructed from ARPES data can be found in the Supplemental Material SI D [31].

Interestingly, we have obtained various values of CDW gaps $2 \Delta$ varying from below $50 \mathrm{meV}$ [e.g., the gap between $\mathrm{G}$ and $\mathrm{H}$ shown in Fig. 6(c)] to above $140 \mathrm{meV}$ [e.g., the gap near L and Q shown in Figs. 6(d) and 6(e), respectively] at different energy-momentum positions and noticed that the magnitude of CDW gap has a strong dependence on nesting degeneracy (which is defined by the number of the first order FBs intersected with the given MB). This effect is most evident along gap J-L where the magnitude jumps from about 80 to over $140 \mathrm{meV}$ near the quadruple L point [see Fig. 6(d)]. The degeneracy dependence argument also provides an explanation for the anisotropy of the gap size on the $\overline{\mathrm{K}}$-centered hole-pocket reported in the early study [10]. Since point $E$ at $\bar{K}-\bar{\Gamma}$ direction is also quadruple-degenerated, gap size reaches its maxima accordingly [see Figs. 5(f) and 6(b)]. Furthermore, as the concept of a constant gap value is no longer valid, we can put an end to debates among various experimental results from 24 to $160 \mathrm{meV}$ [4].

Our TB model shows that most of the experimentally observed CDW gaps have a nesting degeneracy factor of 2 except for the highly degenerate crossing points of gaps [e.g., A-B-C not including B and C shown in Fig. 5(e), D-E not including $\mathrm{E}$ shown in Fig. 5(f), and I-J-L not including $\mathrm{J}$ and $\mathrm{L}$ shown in Fig. 5(g)]. The magnitude of these band gap with a nesting degeneracy factor of 2 range from 40 to $90 \mathrm{meV}$ [see Fig. 6]. The anisotropy in gap magnitude with the same nesting degeneracy suggests that a moderate-to-strong momentumdependent electron-phonon coupling $[17,19,20,35]$ might also play a role in the formation of CDW.

Although the CDW gaps below $E_{F}$ might have limited contribution to the energy gain of electron subsystem and thus attract less research interest, we prove that they can be the key to 
(a) Hole Pocket @ $\bar{\Gamma}$

(b)

Hole Pocket @ K

(c)

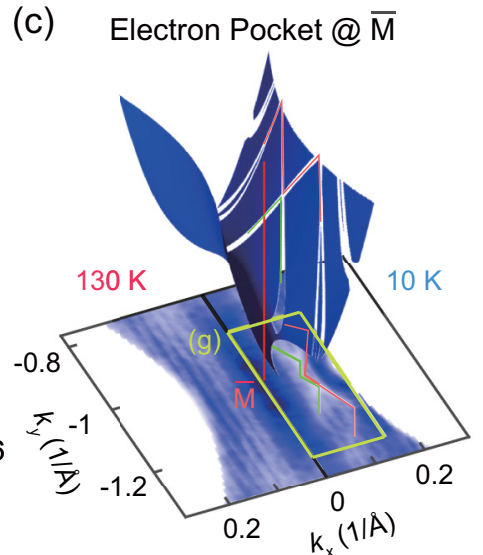

(d)
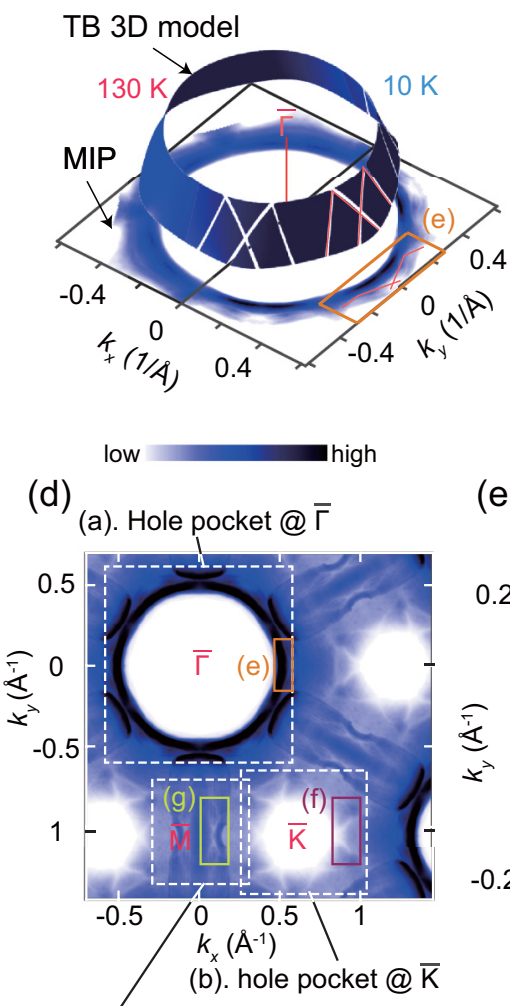

(c). Electron pocket @ $\bar{M}$
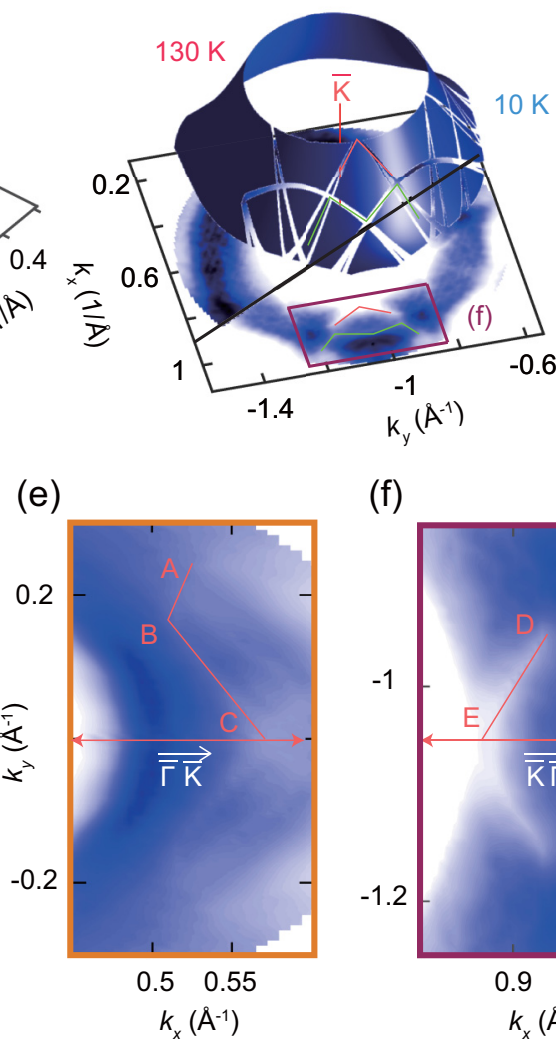

(f)

(g)

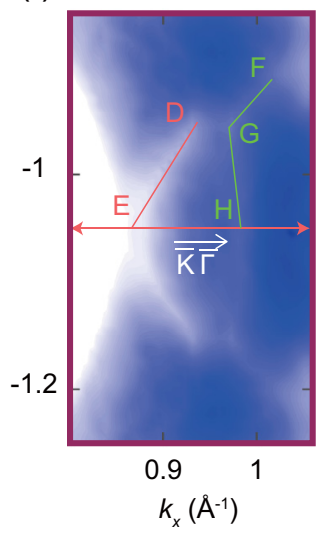

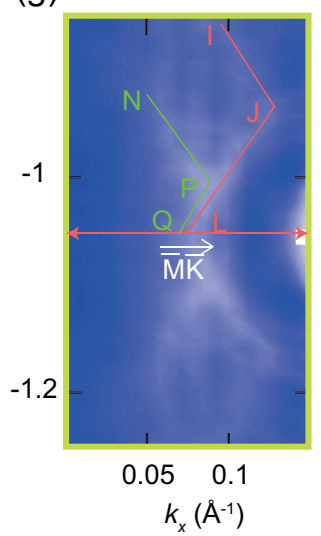

FIG. 5. Maximum intensity projection (MIP) and CDW gap candidates. (a)-(c) Illustration of 3D TB band sheet (upper) and corresponding MIP map (lower) in three momentum area: hole-pocket centered at $\bar{\Gamma}$ (a), hole-pocket centered at $\overline{\mathrm{K}}$ (b), and dog-bone electron-pocket centered at $\bar{M}(\mathrm{c})$. The transparent patterns in the 3D models are intersection lines between the MB sheet (blue surface) and the first-order FB sheets (not shown), which are regarded as CDW gap candidates. Their projection matches nicely with the dip features in MIP. (d) MIP map in a wide momentum range. White dashed rectangles indicate the momentum areas shown in (a)-(c) and colored rectangles indicate the momentum areas shown in (e)-(g). (e)-(g) Zoomed in MIP map of specific momentum area in (a)-(c). The projection of the CDW gap candidates in $k_{x}-k_{y}$ plane is highlighted by solid lines.

understand the CDW formation in the quasi-2D TMD system. First, as the gaps at $E_{F}$ are extensions of the lower ones, they must have the same origin which we attribute to the hybridization between MBs and FBs. Second, the magnitudes of gaps below $E_{F}$ are well defined as both the lower and upper bands are clearly shown in ARPES measurement. The magnitudes of gaps at $E_{F}$, on the contrary, are tricky to quantitatively measure as the upper conduction band is unreachable. To guess the full gap size, the assumption of particle-hole symmetry is applied, which might not be preserved in this system [36]. Since the gaps at and below $E_{F}$ are evolved from one to another and have the same nature, they should have comparable size. Therefore, surprisingly, since CDW formation results in prominent gap opening at high binding energy, it might imply that band sheets below $E_{F}$ also play a part in the transition in contrast to the conventional FS nesting [32].

\section{DISCUSSION AND CONCLUSION}

Our hybridization interpretation on CDW gaps in $2 \mathrm{H}-\mathrm{TaSe}_{2}$ based on the fine band structure detected by ARPES is a natural expansion and development of previous ARPES studies $[4,10]$. Rossnagel et al. [10] have proposed that band hybridization can be an alternative explanation for the high-energy kinks and anisotropy gap around $\mathrm{K}$ compared to the electron-boson mode coupling effect argument. In this paper, by mapping out the CDW gap distribution over the whole BZ, we recognize that the high energy "kinks" are extension of CDW gaps from Fermi level to high binding energy. Our results confirm the validity of band hybridization and provide experimental evidence on momentum-dependent electron-phonon coupling. However, we believe that it is too early to classify this system to strong-coupling regime only based on the unexpected large gap magnitude $[4,10]$. Closer scrutiny should be given to discern effects from dimensionality and coupling strength.

Our analysis on CDW gaps might give an insight on the controversy whether weak-coupling Peierls model [22,23] or strong-coupling McMillan [21] model works better in a realistic quasi-2D system. The experimentally observed band gap magnitude (with a nesting degeneracy factor of 2) of $40-90 \mathrm{meV}$ is significantly larger than predicted value of 37 $\mathrm{meV}$ in weak-coupling limit if the transition temperature of $122 \mathrm{~K}$ is given $\left(2 \Delta(0)=3.52 k_{B} T_{0}\right)$ [4]. On the other hand, the strong-coupling theory provides a reasonable gap size of $73.6 \mathrm{meV}\left(2 \Delta(0)=7 k_{B} T_{0}\right)$ [4]; however, it requires a much severer lattice distortion of $0.16 \AA$ rather than $0.05 \AA$ suggested 



FIG. 6. Band dispersion along CDW gap candidates. Comparison of band dispersion along CDW gap candidates (indicated in Fig. 5) between normal $(130 \mathrm{~K}$, left) and CDW phase (10 K, middle). Energy distribution curves (EDCs) plots (right) at the momentum of interest marked by red and blue arrows in normal and CDW phase, respectively, show a split density of states (DOS) in the low-temperature phase, indicating the formation of CDW gaps.

by neutron scattering measurements [3,21,37]. Our argument on CDW gap formation might give a favorable turn for the classical Peierls model in this dilemma. Peierls classical view in the simple $1 \mathrm{D}$ case is that homogeneous and complete CDW gaps form at the mini-BZ boundary under the interaction be- (a) 1D Case

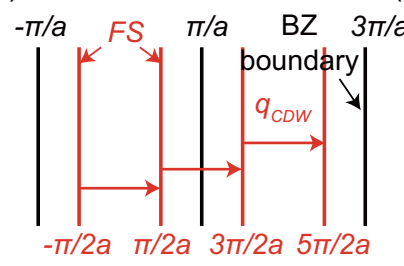

(b)

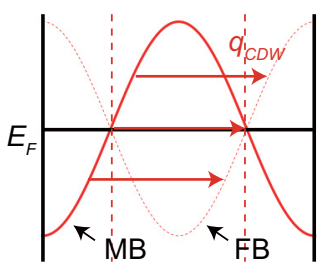

(c)

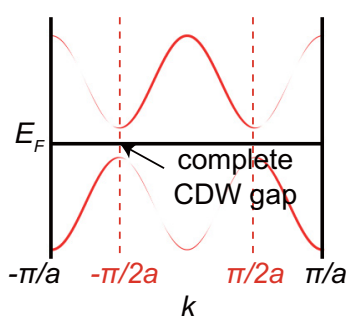

(e)
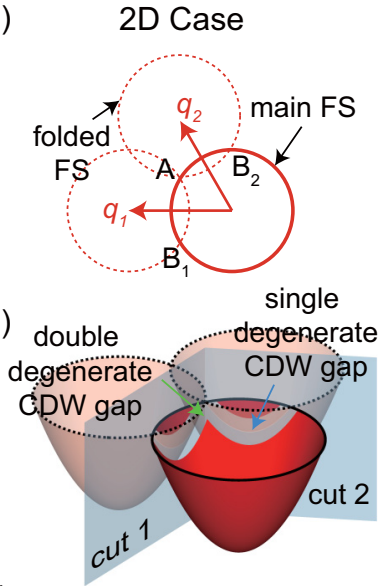

(f) $E$

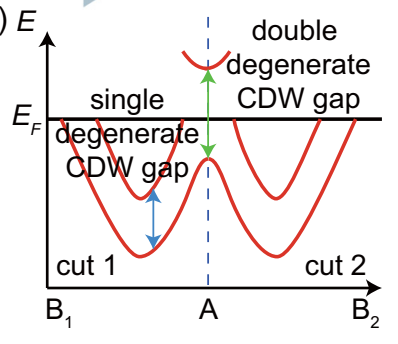

FIG. 7. Schematics of $1 \mathrm{D}$ and 2D Peierls CDW model. (a)-(c) Schematic of 1D Peierls CDW model. FS sheets (a) connected by the CDW nesting vector (marked by red arrows) in the 1D case are parallel to each other. FS nesting condition is well met. Dispersions of the $\mathrm{MB}$ and $\mathrm{FB}$ in the first $\mathrm{BZ}$ cross right at $E_{F}(\mathrm{~b})$. Interaction between the $\mathrm{MB}$ and a single $\mathrm{FB}$ cause the complete CDW gap opening at the boundary of the new mini-BZ (c). (d)-(f) Schematic of 2D Peierls CDW model. In the 2D case, folded FS and main FS only cross at discrete momentum $\left(\mathrm{A}, \mathrm{B}_{1}\right.$, and $\left.\mathrm{B}_{2}\right)$ at $E_{F}(\mathrm{~d})$. Note that two FBs cross with $\mathrm{MB}$ at $\mathrm{A}$. Interaction between the $\mathrm{MB}$ and $\mathrm{FBs}$ only partially create $\mathrm{CDW}$ gaps at $E_{F}$; however, they extend to high binding energy (e). (f) Dispersions along CDW gaps $\mathrm{B}_{1} \mathrm{~A}$ and $\mathrm{AB}_{2}$ indicated by blue shaded cut 1 and 2 in (e). CDW gap magnitude near the double degenerate point $\mathrm{A}$ is significantly larger than neighboring single-degenerate ones. The conduction band near A is not shown in (e).

tween corresponding MB and a single FB [Figs. 7(a)-7(c)]. In contrast, although FS in 2D-CDW system is partially gapped, triple-nesting-vector-induced FBs increase the degeneracy of the band crossing and thus further amplify the band gap magnitude. Degeneracy-enhanced CDW gaps near $E_{\mathrm{F}}$ stabilize the system and compensate for the poor FS nesting condition [Figs. 7(d)-7(f)]. Therefore, although the measured gap size does not match the theoretical prediction, from an "average" perspective, since about half of the FS are gapped by interaction with two FBs, the gap value $\left(2 \Delta \times 0.5 \approx 3.52 k_{B} T_{0}\right)$ should fall into the weak-coupling range.

Finally, taking $2 \mathrm{H}$-TaSe 2 as a canonical example, we describe how CDW are formed and influence the electronic band structure in quasi-2D TMD system. During the transition, the delocalized Ta $5 d$ electrons driven by Peierls instability form a density modulation wave in the real space. Correspondingly in momentum space, FBs are formed as FS nesting suggested. Hybridization between MBs and FBs creates band gaps which 
lower the energy of electron subsystem. Through electronphonon coupling, Ta atoms move to their new equilibrium position and form the $3 \times 3$ super-lattice, whereas Se atoms are barely affected.

In conclusion, we have present a direct observation of the $3 \times 3 \mathrm{CDW}$-induced FBs and constructed an appropriate model that accounts for the CDW gaps in $2 \mathrm{H}-\mathrm{TaSe}_{2}$. Our results and modeling suggest that CDW instability in $2 \mathrm{H}-\mathrm{TaSe}_{2}$ is mainly FS driven and fit into the weak-coupling model; however, electron-phonon coupling has a nonnegligible momentum anisotropy.

\section{ACKNOWLEDGMENTS}

Y.L.C. acknowledges the support from the EPSRC (UK) Platform Grant (Grant No. EP/M020517/1), and National Natural Science Foundation of China (Grant No. 11634009). L.X.Y. acknowledges the support by the NSFC (Grant No. 11774190). Y.W.L. acknowledges the support of the China Scholarship Council-University of Oxford Scholarship. We thank the Diamond Light Source for access to beamline I05 (Proposal SI15364) that contributes to the results presented here. The authors declare no competing financial interests.
[1] C. Scruby, P. Williams, and G. Parry, The role of charge density waves in structural transformations of $1 \mathrm{~T} \mathrm{TaS}_{2}$, Philos. Mag. 31, 255 (1975).

[2] J. A. Wilson, F. Di Salvo, and S. Mahajan, Charge-density waves and superlattices in the metallic layered transition metal dichalcogenides, Adv. Phys. 24, 117 (1975).

[3] D. E. Moncton, J. D. Axe, and F. J. Disalvo, Neutron-scattering study of charge-density wave transitions in $2 \mathrm{H}$-Tase ${ }_{2}$ and 2H-Nbse 2 , Phys. Rev. B 16, 801 (1977).

[4] K. Rossnagel, On the origin of charge-density waves in select layered transition-metal dichalcogenides, J. Phys.: Condens. Matter 23, 213001 (2011).

[5] S. Borisenko, A. Kordyuk, A. Yaresko, V.Zabolotnyy, D. Inosov, R. Schuster, B. Büchner, R. Weber, R. Follath, and L. Patthey, Pseudogap and Charge Density Waves in Two Dimensions, Phys. Rev. Lett. 100, 196402 (2008).

[6] D. Evtushinsky, A. Kordyuk, V. Zabolotnyy, D. Inosov, B. Büchner, H. Berger, L. Patthey, R. Follath, and S. Borisenko, Pseudogap-Driven Sign Reversal of the Hall Effect, Phys. Rev. Lett. 100, 236402 (2008).

[7] D. Inosov, D. Evtushinsky, V. Zabolotnyy, A. Kordyuk, B. Büchner, R. Follath, H. Berger, and S. Borisenko, Temperaturedependent Fermi surface of 2 H-TaSe 2 driven by competing density wave order fluctuations, Phys. Rev. B 79, 125112 (2009).

[8] T. Valla, A. Fedorov, P. Johnson, J. Xue, K. Smith, and F. DiSalvo, Charge-Density-Wave-Induced Modifications to the Quasiparticle Self-Energy in $2 \mathrm{H}-\mathrm{TaSe}_{2}$, Phys. Rev. Lett. 85, 4759 (2000).

[9] D. Rahn, S. Hellmann, M. Kalläne, C. Sohrt, T. Kim, L. Kipp, and $\mathrm{K}$. Rossnagel, Gaps and kinks in the electronic structure of the superconductor $2 \mathrm{H}-\mathrm{NbSe}_{2}$ from angle-resolved photoemission at 1 K, Phys. Rev. B 85, 224532 (2012).

[10] K. Rossnagel, E. Rotenberg, H. Koh, N. V. Smith, and L. Kipp, Fermi surface, charge-density-wave gap, and kinks in $2 \mathrm{H}-\mathrm{TaSe}_{2}$, Phys. Rev. B 72, 121103(R) (2005).

[11] K. Rossnagel, E. Rotenberg, H. Koh, N. V. Smith, and L. Kipp, Continuous Tuning of Electronic Correlations by Alkali Adsorption on Layered 1T-TaS 2 , Phys. Rev. Lett. 95, 126403 (2005).

[12] R. Liu, W. Tonjes, V. Greanya, C. Olson, and R. Frindt, Fermi surface of $2 \mathrm{H}-\mathrm{TaSe}_{2}$ and its relation to the charge-density wave, Phys. Rev. B 61, 5212 (2000).

[13] R. Liu, C. Olson, W. Tonjes, and R. Frindt, Momentum Dependent Spectral Changes Induced by the Charge Density Wave in $2 \mathrm{H}-\mathrm{TaSe}_{2}$ and the Implication on the CDW Mechanism, Phys. Rev. Lett. 80, 5762 (1998).
[14] C. Wang, B. Giambattista, C. Slough, R. Coleman, and M. Subramanian, Energy gaps measured by scanning tunneling microscopy, Phys. Rev. B 42, 8890 (1990).

[15] J. Demsar, L. Forró, H. Berger, and D. Mihailovic, Femtosecond snapshots of gap-forming charge-density-wave correlations in quasi-two-dimensional dichalcogenides $1 \mathrm{~T}-\mathrm{TaS}_{2}$ and $2 \mathrm{H}-\mathrm{TaSe}_{2}$, Phys. Rev. B 66, 041101 (2002).

[16] D. Inosov, V. Zabolotnyy, D. Evtushinsky, A. Kordyuk, B. Büchner, R. Follath, H. Berger, and S. Borisenko, Fermi surface nesting in several transition metal dichalcogenides, New J. Phys. 10, 125027 (2008).

[17] M. Johannes and I. Mazin, Fermi surface nesting and the origin of charge density waves in metals, Phys. Rev. B 77, 165135 (2008).

[18] G. Gruner, The dynamics of charge-density waves, Rev. Mod. Phys. 60, 1129 (1988).

[19] Y. Yoshida and K. Motizuki, Electron-lattice interactions and lattice instabilities of $1 \mathrm{~T}-\mathrm{VSe}_{2}, 1 \mathrm{~T}-\mathrm{CrSe} \mathrm{S}_{2}$, and $1 \mathrm{~T}-\mathrm{TiS}_{2}$, J. Phys. Soc. Jpn. 51, 2107 (1982).

[20] K. Motizuki and E. Ando, Theoretical-study of lattice instability and lattice-dynamics of $2 \mathrm{H}-\mathrm{TaSe}_{2}$ and $2 \mathrm{H}-\mathrm{NbSe}_{2}$ : Electronlattice interaction and generalized electronic susceptibility, J. Phys. Soc. Jpn. 52, 2849 (1983).

[21] W. L. McMillan, Microscopic model of charge-density waves in 2H-TaSe 2 , Phys. Rev. B 16, 643 (1977).

[22] S.-K. Chan and V. Heine, Spin density wave and soft phonon mode from nesting Fermi surfaces, J. Phys. F 3, 795 (1973).

[23] A. W. Overhauser, Charge-density waves and isotropic metals, Adv. Phys. 27, 343 (1978).

[24] D. Shen, B. Xie, J. Zhao, L. Yang, L. Fang, J. Shi, R. He, D. Lu, H. Wen, and D. Feng, Novel Mechanism of a Charge Density Wave in a Transition Metal Dichalcogenide, Phys. Rev. Lett. 99, 216404 (2007).

[25] T. M. Rice and G. K. Scott, New Mechanism for a Charge-Density-Wave Instability, Phys. Rev. Lett. 35, 120 (1975).

[26] J. X. Dai, E. Calleja, J. Alldredge, X. D. Zhu, L. J. Li, W. J. Lu, Y. P. Sun, T. Wolf, H. Berger, and K. McElroy, Microscopic evidence for strong periodic lattice distortion in two-dimensional charge-density wave systems, Phys. Rev. B 89, 165140 (2014).

[27] A. H. Castro Neto, Charge Density Wave, Superconductivity, and Anomalous Metallic Behavior in 2D Transition Metal Dichalcogenides, Phys. Rev. Lett. 86, 4382 (2001). 
[28] P. Leininger, D. Chernyshov, A. Bosak, H. Berger, and D. Inosov, Competing charge density waves and temperature-dependent nesting in $2 \mathrm{H}-\mathrm{TaSe}_{2}$, Phys. Rev. B 83, 233101 (2011).

[29] A. König, K. Koepernik, R. Schuster, R. Kraus, M. Knupfer, B. Büchner, and H. Berger, Plasmon evolution and chargedensity wave suppression in potassium intercalated $2 \mathrm{H}-\mathrm{TaSe}_{2}$, Europhys. Lett. 100, 27002 (2012).

[30] K. Rossnagel and N. Smith, Spin-orbit splitting, Fermi surface topology, and charge-density-wave gapping in $2 \mathrm{H}-\mathrm{TaSe}_{2}$, Phys. Rev. B 76, 073102 (2007).

[31] See Supplemental Material at http://link.aps.org/supplemental/ 10.1103/PhysRevB.97.115118 for more detailed ARPES data, tight-binding fitting model and illustration of MIP method.

[32] J. Laverock, D. Newby, Jr., E. Abreu, R. Averitt, K. Smith, R. Singh, G. Balakrishnan, J. Adell, and T. Balasubramanian, $k$-resolved susceptibility function of $2 \mathrm{H}-\mathrm{TaSe}_{2}$ from angle-resolved photoemission, Phys. Rev. B 88, 035108 (2013).
[33] M. Chhowalla, H. S. Shin, G. Eda, L. J. Li, K. P. Loh, and H. Zhang, The chemistry of two-dimensional layered transition metal dichalcogenide nanosheets, Nat. Chem. 5, 263 (2013).

[34] R. A. Craven and S. F. Meyer, Specific-heat and resistivity near charge-density-wave phase-transitions in $2 \mathrm{H}-\mathrm{TaSe}_{2}$ and 2H-TaS 2 , Phys. Rev. B 16, 4583 (1977).

[35] Y. Ge and A. Y. Liu, Effect of dimensionality and spin-orbit coupling on charge-density-wave transition in $2 \mathrm{H}-\mathrm{TaSe}_{2}$, Phys. Rev. B 86, 104101 (2012)

[36] A. Soumyanarayanan, M. M. Yee, Y. He, J. van Wezel, D. J. Rahn, K. Rossnagel, E. W. Hudson, M. R. Norman, and J. E. Hoffman, Quantum phase transition from triangular to stripe charge order in $\mathrm{NbSe}_{2}$, Proc. Natl. Acad. Sci. U.S.A. 110, 1623 (2013).

[37] R. L. Withers and L. A. Bursill, Comments on the neutronscattering study of the charge-density-wave- periodic-structuraldistortion transition in $2 \mathrm{H}-\mathrm{Tase}_{2}$ and $2 \mathrm{H}-\mathrm{Nbse}_{2}$ by Moncton, Axe, and Disalvo, Phys. Rev. B 26, 1469 (1982). 